\title{
Second Microlocalization and the Mellin Transformation
}

\author{
By
}

\author{
Bogdan Ziemian* and Henryk KoŁAKowski**
}

\section{§1. Introduction}

Let a conormal distribution $u$ be a solution of the equation

$$
\frac{\partial^{2} u}{\partial t^{2}}-\frac{\partial^{2} u}{\partial x^{2}}-\frac{\partial^{2} u}{\partial y^{2}}=f(t, x, y, u)
$$

in an open set $\Omega \subset \boldsymbol{R}^{3}$ such that $(t, x, y)=(0,0,0) \in \Omega$.

In paper [1] J.M. Bony showed that if for $t<0 u$ had conormal singularities situated on the characteristic surfaces $\Sigma_{1}, \Sigma_{2}, \Sigma_{3}$ then for $t>0 u$ is regular outside $\Sigma_{1}, \Sigma_{2}, \Sigma_{3}$ and the forward light cone starting at the point $\Sigma_{1} \cap \Sigma_{2} \cap \Sigma_{3}$.

A basic tool used in the proof of this fact was, among other things, the notion of second microlocalization and in particular of second wave front set.

As it is well known the notion of the first wave front set WFu of a distribution $u$ can be described with the help of the Fourier transformation. We show that in the case of the second wave front set an analogous role is played by the Mellin transformation. Moreover we give a new proof of Bony's theorem on propagation of 2-microlocal singularities.

\section{Second Wave Front Set in Terms of the Space $\operatorname{SP}\left(s, s^{\prime}\right)$}

Definition 1. (cf. Def. 2.5 [1]). Let $u \in D^{\prime}\left(\boldsymbol{R}^{n} \backslash\{0\}\right)$ and let $u$ vanish outside a unit ball in $\boldsymbol{R}^{n}$. Let $s, s^{\prime} \in \boldsymbol{R}$ be such that $s+s^{\prime}$ is a non-negative integer i.e. $s+s^{\prime} \in N_{0}$. We say that $u \in S P\left(s, s^{\prime}\right)$ if

Communicated by M. Kashiwara, August 8, 1988.

* Institute of Mathematics, Polish Academy of Sciences Sniadeckich 8, 00-950 Warsaw, Poland.

** Department of Mathematics of University of Warsaw, PKiN, IXp. 00-901 Warsaw, Poland. 


$$
\begin{aligned}
& \|x\|^{-s+|\lambda|} D^{\lambda} u \in L^{2}\left(\mathbb{R}^{n}\right) \quad \text { for } \quad 0 \leq|\lambda| \leq s+s^{\prime} \\
& \lambda=\left(\lambda_{1}, \lambda_{2}, \cdots, \lambda_{n}\right) \in \mathbb{N}_{0}^{n}, \quad|\lambda|=\lambda_{1}+\lambda_{2}+\cdots+\lambda_{n} .
\end{aligned}
$$

For remaining $\left(s, s^{\prime}\right)$ the spaces $\operatorname{SP}\left(s, s^{\prime}\right)$ are defined by duality and interpolation.

Let for $\tau \in \mathbb{R}^{n}, x \in \mathbb{R}_{+}^{n}=\left\{x \in \mathbb{R}^{n}: x_{i}>0 i=1, \cdots, n\right\} x^{\tau}=x_{1}^{\tau} \cdots \cdots x_{n}^{\tau}$. The point $(a, \cdots, a) \in \mathbb{R}^{n}$ we denote by bold face $a$.

In our case it will be more convenient to use, instead of the space $L^{2}\left(\boldsymbol{R}^{n}\right)$, the space $L^{2}\left(\mathbb{R}_{+}^{n}\right)$ with the weight $x^{-1}$ i.e.

$$
u \in L^{2}\left(\boldsymbol{R}_{+}^{n}, x^{-1}\right) \text { if and only if } \int_{\boldsymbol{R}_{+}^{n}}|u(x)|^{2} x^{-1} d x<\infty .
$$

For $u \in D^{\prime}\left(\mathbb{R}_{+}^{n}\right)$, (1) can be written as $u \in S P\left(s, s^{\prime}\right)$ if and only if

$$
x^{1 / 2}\|x\|^{-s+|\lambda|} D^{\lambda} u \in L^{2}\left(\boldsymbol{R}_{+}^{n}, x^{-1}\right), \quad 0 \leq|\lambda| \leq s+s^{\prime} .
$$

If supp $u \subset \Gamma$, where $\Gamma$ is a proper cone in $\mathbb{R}_{+}^{n}$ (i.e. such that $\bar{\Gamma} \cap \overline{\mathbb{R}_{+}^{n}}=\{0\}$, overbar denotes closure in $\mathbb{R}^{n}$ ) then there exist constants $c_{j}, d_{j}>0$ such that $c_{j} x_{j}<\|x\|<d_{k} x_{k}, j, k=1,2, \cdots, n$ for $x \in \Gamma$ (shortly $\left.\forall_{k} x_{k} \sim\|x\|\right)$. Then (2) denotes that

$$
x^{1 / 2-\rho}(x D)^{\lambda} u \in L^{2}\left(\mathbb{R}_{+}^{n}, x^{-1}\right)
$$

for $\lambda \in \mathbb{N}_{0}^{n}, \rho \in \mathbb{R}^{n}, 0 \leq|\lambda| \leq s+s^{\prime},|\rho|=\rho_{1}+\cdots+\rho_{n}=s$ where $(x D)^{\lambda}=\left(x_{1} D_{1}\right)^{\lambda_{1}} \cdots\left(x_{n} D_{n}\right)^{\lambda_{n}}$, i.e. that

$$
x^{\gamma}(x D)^{\lambda} u \in L^{2}\left(\mathbb{R}_{+}^{n}, x^{-1}\right) \quad \text { for } \quad \lambda \in \mathbb{N}_{0}^{n}, r \in \mathbb{R}^{n},|\lambda| \leq s+s^{\prime}
$$

where $|r|=r_{1}+\cdots+r_{n} \geq-s+\frac{n}{2}$, which is equivalent to

$$
x^{\gamma}(x D)^{\lambda} u \in L^{2}\left(\mathbb{R}_{+}^{n}, x^{-1}\right) \quad \text { for } \quad \lambda \in \mathbb{N}_{0}^{n},|\lambda| \leq s+s^{\prime}
$$

and some $r_{0} \in \mathbb{R}^{n}$ such that $\left|r_{0}\right|=-s+\frac{n}{2}$.

Let $\Gamma \subset \boldsymbol{R}_{+}^{n}$ be a cone tangent only to the axis $x_{1}$ i.e. such that closure $\bar{\Gamma} \cap\left\{x \in \boldsymbol{R}^{n}: x_{1}=0\right\}=\{0\}$. Then we have $\|x\| \sim x_{1}$ on $\bar{\Gamma}$ thus the condition

$$
x^{1 / 2}\|x\|^{-s+|\lambda|} D^{\lambda} u \in L^{2}\left(\Gamma, x^{-1}\right), \quad|\lambda| \leq s+s^{\prime}
$$

is equivalent to the condition

$$
x^{1 / 2} x_{1}^{-s+|\lambda|} D^{\lambda} u \in L^{2}\left(\Gamma, x^{-1}\right), \quad|\lambda| \leq s+s^{\prime} .
$$

Fix $\delta \dot{x}=\left(\delta \dot{\circ}_{1}, \cdots, \delta \dot{x}_{n}\right) \in \mathbb{R}_{+}^{n}, \stackrel{\dot{\xi}}{=}\left(\dot{\circ}_{1}, \cdots, \dot{\circ}_{n}\right) \in \mathbb{R}^{n}$ and let 
$1^{\circ} \rho \in C^{\infty}\left(\mathrm{i}\left(\boldsymbol{R}^{n} \backslash\{0\}\right)\right)$ be a homogeneous function of order zero defined in a

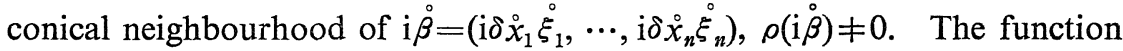
$\rho$ is extended to the set $\boldsymbol{R}^{n}+\mathrm{i}\left(\boldsymbol{R}^{n} \backslash\{0\}\right)$ by putting $\rho(z)=\rho(\operatorname{Im} z)$.

$2^{\circ} \boldsymbol{\kappa}=\varphi \cdot \boldsymbol{\kappa}^{\prime}$ where $\varphi$ is a $C_{0}^{\infty}$ bump function at zero and $\boldsymbol{\kappa}^{\prime} \in C^{\infty}\left(\boldsymbol{R}^{n} \backslash\{0\}\right)$ is a cut-off function in a conical neighbourhood of $\delta \dot{x}$ i.e. supp $\boldsymbol{\kappa}^{\prime} \subset \boldsymbol{R}_{+}^{n}, \boldsymbol{\kappa}^{\prime}$ is homogeneous of order zero and $\kappa^{\prime}\left(\delta x^{\circ}\right) \neq 0$.

$3^{\circ} \chi \in C^{\infty}\left(\mathrm{i}\left(\boldsymbol{R}^{n} \backslash\{0\}\right)\right)$ be a function with supp $\chi \subset\{\mathrm{i} \tau: 1 / 4 \leq\|\tau\|\}$ and $\chi(\mathrm{i} \tau)$ $\equiv 1$ for $\tau \in\{\tau: 1 / 2 \leq\|\tau\|\}$. The function $\chi$ is extended to $\boldsymbol{R}^{n}+\mathrm{i}\left(\boldsymbol{R}^{n} \backslash\{0\}\right)$ by putting $\chi(z)=\chi(\operatorname{Im} z)$.

Definition 2. Let $(\stackrel{\circ}{x}, \stackrel{\circ}{\xi}) \in T_{0}^{*}\left(\boldsymbol{R}^{n}\right) \simeq\left\{(0, \xi): \xi \in \boldsymbol{R}^{n}\right\}, \delta \stackrel{0}{x} \in \boldsymbol{R}_{+}^{n}, \delta \stackrel{\circ}{\xi} \in \boldsymbol{R}^{n}$, $u \in S P(s,-\infty)=\bigcup_{\sigma^{\prime}} S P\left(s, \sigma^{\prime}\right)$. We say that $u$ belongs to $S P\left(s, s^{\prime}\right)$ 2-microlocally at the point $(\stackrel{\circ}{x}, \stackrel{\circ}{\xi}, \delta \dot{x}, \delta \dot{\xi})=(0, \dot{\xi}, \delta \dot{x}, 0)$ if there exist functions $\rho, k, \chi$ satisfying conditions $1^{\circ}, 2^{\circ}, 3^{\circ}$ respectively, such that

$$
\widetilde{P}(x, D) u=\chi(x D) \rho(x D) \kappa(x) u \in S P\left(s, s^{\prime}\right) .
$$

If $u \in S P(s,-\infty)$ we define its $S P\left(s, s^{\prime}\right)$-second wave front set (denoted $\left.2 \mathrm{WF}^{S P\left(s, s^{\prime}\right)} u\right)$ as a closed subset of the space

$$
\left(T^{*}\left(\boldsymbol{R}^{n}\right) \backslash T_{0}^{*}\left(\boldsymbol{R}^{n}\right)\right) \cup N_{T_{0}^{*}\left(\boldsymbol{R}^{n}\right)}\left(T\left(T^{*} \boldsymbol{R}^{n}\right)\right)
$$

consisting of the points $(x, \xi) \notin T_{0}^{*}$ such that $u \notin S P\left(s+s^{\prime}, 0\right)$ microlocally at the point $(x, \xi)$ and of the points $(0, \xi, \delta x, 0)$ such that $u \notin S P\left(s, s^{\prime}\right) 2$-microlocally (note that in our case the space normal to $T_{0}^{*}\left(\boldsymbol{R}^{n}\right)$ at the point $(\stackrel{\circ}{x}, \stackrel{\circ}{\xi})$ can be identified with the set of vectors of the form $(\delta \dot{x}, 0)$ with $\delta \dot{x} \in \boldsymbol{R}^{n}$ and the topology in $N_{T_{0}^{*}\left(R^{n}\right)}$ coincides with the topology of conical neighbourhoods of the vectors $\delta \dot{x}$ in $\boldsymbol{R}^{n}$ ).

Definition 3 ([1]). We say that a function $a(x, \xi) \in C^{\infty}\left(\boldsymbol{R}^{2 n}\right)$ belongs to $\sum_{0}^{m, m^{\prime}}$ if and only if

$$
\left|D_{\xi}^{\alpha} D_{x}^{\beta} a(x, \xi)\right| \leq C_{\alpha \beta}\langle\xi\rangle^{m-|\alpha|+\beta \mid}(1+\|x\| \| \xi||)^{m^{\prime}-|\beta|}
$$

where $\langle\xi\rangle=\left(1+\|\xi\|^{2}\right)^{1 / 2}$.

Definition 4 ([1]). We say that a function $a(x, \xi) \in C^{\infty}\left(\left(\boldsymbol{R}^{n} \backslash\{0\} \times \boldsymbol{R}^{n}\right)\right.$ belongs to $S \sum_{0}^{m, m^{\prime}}$ if

a) $a(x, \xi)$ is flat in the following sense:

$$
\left|D_{\xi}^{\alpha} D_{x}^{\beta} a(x, \xi)\right| \leq C_{\alpha \beta}\|x\|^{-m+|\alpha|-|\beta|}(1+\| x|||\xi| \mid)^{m+m^{\prime}-|\alpha|}
$$

b) $a(x, D)$ is properly supported, in a strong sense, in $\boldsymbol{R}^{n} \backslash\{0\}$ i.e. 


$$
\operatorname{supp} \hat{a}^{2}(x, x-y) \subset\left\{(x, y): k^{-1}<\frac{\|y\|}{\|x\|}<k\right\}
$$

for some $k>1$. Here, $\hat{a}^{2}$ means the Fourier transform with respect to $\xi$. As in [1] for the function $a(x, \xi) \in \sum_{0}^{m, m^{\prime}}\left(S \sum_{0}^{m, m^{\prime}}\right)$ we define an operator $a(x, D) \in$ $\mathrm{Op}\left(\sum_{0}^{m, m^{\prime}}\right)\left(\mathrm{Op}\left(S \sum_{0}^{m, m^{\prime}}\right)\right)$.

Definition 5 ([1]). Let $\Lambda$ be a conic lagrangean submanifold of $T^{*} \mathbb{R}^{n}$ (here $\Lambda$ means $T_{0}^{*}\left(\mathbb{R}^{n}\right)$ ). Let $(\stackrel{\circ}{\dot{x}}, \stackrel{\circ}{\xi})^{\circ}$ belong to $\Lambda$ and let $(\delta \dot{x}, \delta \dot{\xi})$ be a vector tangent to $T^{*} \mathbb{R}^{n}$ at that point $(\dot{x}, \dot{\xi})$ and not tangent to $\Lambda$ at that point. If $A \in \mathrm{Op}\left(\sum_{0}^{m, m^{\prime}}\right)$, we will say that $A$ is elliptic at $(\stackrel{\circ}{x}, \stackrel{\circ}{\xi}, \delta \dot{x}, \delta \xi)$ if one has

$$
|a(\dot{x}+\varepsilon \delta \dot{x}, \lambda(\dot{\xi}+\varepsilon \delta \dot{\xi}))| \geq C \lambda^{m}(\lambda \varepsilon)^{m^{\prime}}
$$

with $C>0$, for $0<\varepsilon \leq \varepsilon_{0}$ and $\lambda \varepsilon>\mu_{0}>0(A=a(x, D))$.

Definition $6([1])$. Let $u \in D^{\prime}\left(\mathbb{R}^{n}\right)$ and let $u$ vanish outside a unit ball in $\boldsymbol{R}^{n}$. We say that $u \in H^{s, k}\left(s \in \mathbb{R}, k \in \mathbb{N}_{0}\right)$ if

$$
x^{\alpha} u \in H^{s+|\alpha|}\left(\mathbb{R}^{n}\right) \quad \text { for } \quad \alpha \in \mathbb{N}_{0}^{n},|\alpha| \leq k \text {. }
$$

For remaining $\left(s, s^{\prime}\right)$ the spaces $H^{s, s^{\prime}}$ are defined by duality and interpolation.

Let

$$
\begin{gathered}
\sum_{0}^{m,-\infty}=\bigcap_{m^{\prime}} \sum_{0}^{m, m^{\prime}} \quad S \sum_{0}^{m,-\infty}=\bigcap_{m^{\prime}} S \sum_{0}^{m, m^{\prime}} \\
H^{s,+\infty}=\bigcap_{s^{\prime}} H^{s, s^{\prime}} \quad S P(s,+\infty)=\bigcap_{s^{\prime}} S P\left(s, s^{\prime}\right) \\
S P(s,-\infty)=\bigcup_{s^{\prime}} S P\left(s, s^{\prime}\right) \quad S P(-\infty,-\infty)=\bigcup_{s, s^{\prime}} S P\left(s, s^{\prime}\right)
\end{gathered}
$$

Lemma 1. Let $\delta \dot{x} \in \mathbb{R}_{+}^{n},\|\delta \dot{x}\|=1, \dot{\xi} \in \mathbb{R}^{n}$. Let $x, \rho, \kappa$ be defined as in $1^{\circ}-3^{\circ}$ and $x \xi=\left(x_{1} \xi_{1}, \cdots, x_{n} \xi_{n}\right)$ then

$$
\widetilde{P}(x, \xi)=\chi(\mathrm{i} x \xi) \circ(\mathrm{i} x \xi) \kappa(x)
$$

belongs to $S \sum_{0}^{0,0}$ (actually to $\sum_{0}^{0,0}$, and the corresponding operator in $\mathrm{Op}\left(\Sigma_{0}^{0,0}\right)$ is elliptic at the point $(0, \dot{\xi}, \delta \dot{x}, 0))$. Moreover the symbol $\widetilde{P}(x, \xi)$ is concentrated around $\left(0, \dot{\xi}, \delta x^{\circ}\right)$ if and only if

$$
P(x, z)=\chi(z) \rho(z) \kappa(x)
$$

is concentrated around $(0, \mathrm{i} \dot{\beta}, \delta \dot{x})$ where $\mathrm{i} \dot{\beta}=\left(\mathrm{i} \delta \dot{x}_{1} \dot{\xi}_{1}, \cdots, \mathrm{i} \delta \dot{x}_{n} \dot{\xi}_{n}\right)$.

Proof. We show the second part of the lemma. Since $\rho$ is homogeneous of order 0 we have 


$$
\rho(\mathrm{i} x \xi)=\rho\left(\mathrm{i} \frac{x}{\|x\|} \xi\right)
$$

$\kappa$ is concentrated around $\delta \dot{x}$ thus on the support of $\kappa$ we have that $\frac{x}{\|x\|}$ is close to $\delta \dot{x}$. On the support of $\rho$ we have that $\mathrm{i} \frac{x}{\|x\|} \xi$ is close to $\mathrm{i} \dot{\beta}$. Since for $\frac{x}{\|x\|}$ close to $\delta \circ, \frac{x}{\|x\|} \in \boldsymbol{R}_{+}^{n}$ it follows from the continuity of the operation of division that $\xi$ is close to $\stackrel{\circ}{\xi}$ on $\operatorname{supp} \widetilde{P}(x, \xi)$.

Proposition 1. $S P\left(s, s^{\prime}\right)$-second wave front set and $H^{s, s^{\prime}}$-second wave front set defined by Bony coincide. More precisely: Let $g$ be a smooth function with support in the ball $B(0,1) \subset \boldsymbol{R}$ equal to zero in a neighbourhood of zero. Then (see Remark 2.10, [1]) the mapping

$$
S P\left(s, s^{\prime}\right) \ni u \stackrel{\Pi}{\longrightarrow} g(\|x\|\|D\|) u \in H^{s, s^{\prime}}
$$

where $g(\|x\||| D||) u=\frac{1}{(2 \pi \mathrm{i})^{n}} \int e^{\mathrm{i}(x, \xi)} g(\| x|||| \xi||) \hat{u}(\xi) d \xi$

defines an isomorphism $\Pi$ of the spaces

$$
S P\left(s, s^{\prime}\right) / S P(s, \infty) \stackrel{\Pi}{\longrightarrow} H^{s, s^{\prime}} / H^{s, \infty} .
$$

Second wave front sets are well defined on the quotient space, thus we have well defined mappings

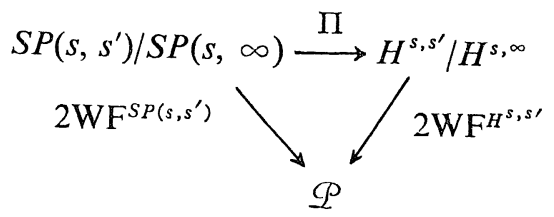

where $\mathscr{P}$ is the set of closed subsets of the space

$$
\left(T^{*} \boldsymbol{R}^{n} \backslash T_{0}^{*}\left(\boldsymbol{R}^{n}\right)\right) \cup N_{T_{0}^{*}\left(\boldsymbol{R}^{n}\right)}\left(T\left(T^{*} \boldsymbol{R}^{n}\right)\right),
$$

and $2 \mathrm{WF}^{B}$ means $B$-second wave front set.

Assertion: The diagram (6) commutes.

Proof. We shall prove that if $u \in S P\left(s, s^{\prime}\right) 2$-microlocally at the point $(\hat{x}, \stackrel{\circ}{\xi}, \delta \stackrel{x}{x}, 0)$ where $(\stackrel{\circ}{x}, \stackrel{\circ}{\xi}) \in T_{0}^{*}\left(\boldsymbol{R}^{n}\right)$ then $\Pi u=g(\|x\|\|D\|) u \in H^{s, s^{\prime}}$ 2-microlocally at that point. Let $P f^{*}=\Pi$, then $P f$ induces an isomorphism $P f$ inverse to $\Pi$. According to Definition 2 we have $\widetilde{P} u \in S P\left(s, s^{\prime}\right)$ hence 


$$
\widetilde{P}(P f \Pi u) \in S P\left(s, s^{\prime}\right)
$$

because $P f \Pi u-u \in S P(s, \infty)$ (Th. 29, [1]) and $\widetilde{P}(x, D) \in \mathrm{Op}\left(S \sum_{0^{0}, 0}\right)$. It follows from (7) that

$$
(\Pi \widetilde{P} P f) \Pi u \in H^{s, s^{\prime}} \text {. }
$$

It is clear that $\Pi \widetilde{P} P f$ is an operator in $\operatorname{Op}\left(\Sigma_{0}^{0,0}\right)$ (for $\widetilde{P}$ is an operator in $\left.\mathrm{Op}\left(S \sum_{0}^{0,0}\right)\right)$. We still have to check that $\Pi \widetilde{P} P f$ is 2 -microelliptic at $(\stackrel{\circ}{x}, \stackrel{\circ}{\xi}, \delta \stackrel{\circ}{x}, 0)$. But this is clear since the principal symbol

$$
\sigma(\Pi \tilde{P} P f)=\chi(\mathrm{i} x \xi) \tau(\mathrm{i} x \xi) \kappa(x) g(\|x\|\|\xi\|)=\widetilde{P}(x, \xi) g(\|x\|\|\xi\|) .
$$

(By Definition in [1] $\sigma(\Pi \widetilde{P} P f)$ is equal to the symbol $\sigma(\widetilde{P}) \in S \Sigma_{0}^{0,0}$ after passing through the isomorphism with the space $\sum_{0}^{0,0}$. But $\sigma(\widetilde{P})=\widetilde{P}(x, \xi)$ from the definition of the operator $\widetilde{P} \in O \mathrm{p}\left(S \sum_{0^{0}}^{0,0}\right)$ (Def. 3.5 [1]) since $\widetilde{P}(x, \xi) \in S \sum_{0}^{0,0}$ ). Since $\Pi$ is an isomorphism this ends the proof of commutativity of the diagram (6).

Remark. In Definition 1.9 in [1] of the second wave front set, it can be assumed that the operator $A \in \mathrm{Op}\left(\sum_{0}^{0,0}\right)$ concentrated at $(0, \xi)$ and 2-microelliptic at the point $(0, \stackrel{\circ}{\xi}, \delta \dot{x}, 0)$ is of the form $\Pi \widetilde{P} P f$ where $\widetilde{P}$ is defined by Definition 2 for suitable $\rho, \chi, \kappa$.

Proof. Let $A u \in H^{s, s^{\prime}}$ 2-microlocally. For suitable $\widetilde{P}, \widetilde{P} P f A^{-1} \in \mathrm{Op}\left(\sum_{0}^{0,0}\right)$ and we have $\Pi \widetilde{P} P f A^{-1} A u \in H^{s, s}$. But $A^{-1} A=I+R, R \in O p\left(\sum_{0}^{0,-N}\right)$ hence modulo an operator in $\operatorname{Op}\left(\sum_{0}^{0,-N}\right)$ we have $\Pi \widetilde{P} P f A^{-1} A=\Pi \widetilde{P} P f$.

Our aim is to define the second wave front set in a way analogous to the definition of the (first) wave front set in terms of the growth order of the Fourier transformation. In our case instead of the Fourier transformation we need the Mellin transformation. First we recall the definition of a Mellin distribution [2], [3].

Let

$$
B_{+}=\left\{x \in \mathbb{R}_{+}^{n}: x_{i} \leq 1, \quad i=1, \cdots, n\right\}
$$

Let $a \in \mathbb{R}^{n}$. Denote by $M_{a}=M_{a}\left(B_{+}\right)$the space of functions $\varphi \in C^{\infty}\left(B_{+}\right)$ such that for every $\alpha \in \mathbb{N}_{0}^{n}$

$$
p_{a, \alpha}(\varphi)=\sup _{x \in B_{+}} \mid\left(x^{a+1}(x D)^{\alpha} \varphi \mid<\infty\right.
$$

with topology given by the seminorms $p_{a, a}, \alpha \in \mathbb{N}_{0}^{n}$. Let $\omega \in(\mathbb{R} \cup\{\infty\})^{n}$. We define 


$$
M_{(\omega)}\left(B_{+}\right)=\underset{a<\omega}{\bigcup} M_{a}\left(B_{+}\right) \text {-inductive limit. }
$$

The dual space $M_{(\omega)}^{\prime}\left(B_{+}\right)$is a subspace of distributions and

$$
M^{\prime}\left(B_{+}\right)=\bigcup_{\omega} M_{(\omega)}^{\prime}\left(B_{+}\right)
$$

is called the space of Mellin (transformable) distributions.

Lemma 2. Let $u \in S P\left(s, s^{\prime}\right), s+s^{\prime} \in \boldsymbol{N}_{0}$, supp $u \subset \Gamma$ where $\Gamma$ is a proper cone in $\boldsymbol{R}_{+}^{n}$, then $u \in M_{(\rho)}^{\prime}$ for $\rho \in \boldsymbol{R}^{n}|\rho| \leq s-\frac{n}{2}$.

Proof. From (3) for $\lambda=0$ we have $x^{\gamma} u \in L^{2}\left(\boldsymbol{R}_{+}^{n}, x^{-1}\right)$ for $|r| \geq-s+\frac{n}{2}$ $r \in \boldsymbol{R}^{n}$. Since multiplication by $x^{\gamma}, r \in \boldsymbol{R}^{n}$ maps $M_{(\omega)}^{\prime} \rightarrow M_{(\omega+\gamma)}^{\prime}$ it is enough to show that if $u \in L^{2}\left(\boldsymbol{R}_{+}^{n}, x^{-1}\right)$ then $u \in M_{(0)}^{\prime}$. Let $\varphi \in M_{a}, a<0$; we have for $a<0$

$$
\left|\int_{B_{+}} u \varphi d x\right| \leq \int_{B_{+}}|u|^{2} x^{-1} d x \int_{B_{+}} x^{1}|\varphi|^{2} d x \leq C \int_{B_{+}} x^{-2 a-1} d x<\infty
$$

thus $u$ is a continuous functional on the space $M_{(0)}=\bigcup_{a<0} M_{a}$. If $u \in M_{(\omega)}^{\prime}$ we define the Mellin transform of $u$

$$
(\mathscr{M} u)(z)=u\left[x^{-z-1}\right], \quad \operatorname{Re} z<\omega
$$

(for $z \in \boldsymbol{C}^{n}$ and $\omega \in \boldsymbol{R}^{n}, \operatorname{Re} z<\omega$ means that $\operatorname{Re} z_{j}<\omega_{j} j=1, \cdots, n$ ). The function $(\mathscr{M} u)(z)$ so defined is holomorphic for $\operatorname{Re} z<\omega$.

Lemma 3. $u \in L^{2}\left(\boldsymbol{R}_{+}^{n}, x^{-1}\right)$ if and only if $(\mathscr{M} u)(z) \in L^{2}\left(\boldsymbol{R}^{n}\right)$ as a function of $\operatorname{Im} z$ for each fixed $\operatorname{Re} z \leq 0$ (i.e. $\mathscr{M}(z)$ defined for $\operatorname{Re} z<0$ has a boundary value for $\operatorname{Re} z \rightarrow 0, \operatorname{Re} z<0$ and the boundary value is in $\left.L^{2}\left(\boldsymbol{R}^{n}\right)\right)$.

Proof. The condition $u \in L^{2}\left(\boldsymbol{R}_{+}^{n}, x^{-1}\right)$ is equivalent to the condition that the function $h(y)=u\left(e^{-y}\right) \in L^{2}\left(\mathbb{R}^{n}\right)$. Since

$$
(\mathscr{M u})(z)=\int_{\boldsymbol{R}_{+}^{n}} u\left(e^{-y}\right) e^{(z, y)} d y
$$

we see that the latter condition is equivalent, in view of the Parsevale equality to the condition that $\mathscr{M u}(z) \in L^{2}\left(\boldsymbol{R}^{n}\right)$ for $\operatorname{Re} z=0$, hence also for $\operatorname{Re} z<0$ $\left(u\left(e^{-y}\right) e^{(\operatorname{Re} z, y)}\right.$ is in $L^{2}$ for $\operatorname{Re} z \leq 0$ if $\left.u \in L^{2}\right)$.

From Lemma 3 and the fact that

$$
\left(\mathscr{H}(x D)^{\lambda} u\right)(z)=z^{\lambda} \mathscr{H} u(z)
$$


we get the following characterization of the space $\operatorname{SP}\left(s, s^{\prime}\right)$ :

Corollary $\mathbb{1}$. Let $u \in D^{\prime}\left(B_{+}\right)$, supp $u \subset \Gamma-$ a proper cone, then the following conditions are equivalent:

i) $u \in S P\left(s, s^{\prime}\right), s+s^{\prime} \in \mathbb{N}_{0}$,

ii) $\mathscr{M u} \in \mathcal{O}\left(\left\{z \in \mathbb{C}^{n}: \sum_{j=1}^{n} \operatorname{Re} z_{j}<s-\frac{n}{2}\right\}\right)$

and for every $z \in\left\{z \in \mathbb{C}^{n}: \sum_{j=1}^{n} \operatorname{Re} z_{j} \leq s-\frac{n}{2}\right\}$

$\mathscr{M u}(\operatorname{Re} z+\mathrm{i} \bullet) \in L^{2, s+s^{\prime}}\left(\mathbb{R}^{n}\right)=L^{2}\left(\mathbb{R}^{n},(1+\|\beta\|)^{s+s^{\prime}}\right)$,

iii) $\mathscr{H} u \in \mathcal{O}\left(\left\{z \in \mathbb{C}^{n}: \sum_{j=1}^{n} \operatorname{Re} z_{j}<s-\frac{n}{2}\right\}\right)$

and for some $\stackrel{\circ}{\alpha}$ such that $\sum_{j=1}^{n} \stackrel{\circ}{\alpha}_{j}=s-\frac{n}{2}$

$\mathscr{H} u(\stackrel{\circ}{\alpha}+\mathrm{i} \bullet) \in L^{2, s+s^{\prime}}\left(\mathbb{R}^{n}\right)$.

Now let $\Gamma \subset \mathbb{R}_{+}^{n}$ be a cone tangent only to the axis $x_{1}$.

Proposition 2. Let $u \in D^{\prime}\left(B_{+}\right)$, supp $u \subset \Gamma$-as above. Then the following conditions are equivalent:

i) $u \in L^{2}\left(\Gamma, x^{-1}\right)$,

ii) $\mathscr{M u}(z)$ is holomorphic in the set $\Omega_{0}=\left\{z \in C^{n}: \sum_{j=1}^{n} \operatorname{Re} z_{j}<0, \alpha_{2}<0, \cdots, \alpha_{n}<0\right\}$

and for every $\alpha \in\left\{\alpha \in \mathbb{R}^{n}: \alpha_{1}+\cdots+\alpha_{n} \leq 0, \alpha_{2} \leq 0, \cdots, \alpha_{n} \leq 0\right\}$,

$\mathscr{H u}(\alpha+\mathrm{i} \beta) \in L^{2}\left(\mathbb{R}^{n}\right)$ as a function of $\beta$,

iii) $\mathscr{M u}$ is holomorphic in $\Omega_{0}$ and $\mathscr{M} u(\stackrel{\circ}{\alpha}+\mathrm{i} \beta) \in L^{2}\left(\mathbb{R}^{n}\right)$ for $\stackrel{\circ}{\alpha}=0$.

More generally we have

Proposition 3。 Let $u \in D^{\prime}\left(B_{+}\right)$, supp $u \subset \Gamma$ as above, $s, s^{\prime} \in \mathbb{R}, s+s^{\prime} \in N_{0}$. The following conditions are equivalent:

i) $u \in S P\left(s, s^{\prime}\right)$,

ii) $\mathscr{M u}(z)$ is holomorphic in the set

$$
\begin{aligned}
\Omega_{s, s^{\prime}}=\left\{z \in C^{n}:\right. & \operatorname{Re} z_{1}+\cdots+\operatorname{Re} z_{n}<s-\frac{n}{2}, \\
& \left.\operatorname{Re} z_{2}<s+s^{\prime}-\frac{1}{2}, \cdots, \operatorname{Re} z_{n}<s+s^{\prime}-\frac{1}{2}\right\}
\end{aligned}
$$

and for every $\lambda \in \mathbb{N}_{0}^{n}$ with $|\lambda| \leq s+s^{\prime}$ and $\alpha \in \mathbb{R}^{n}$ such that

$\alpha_{1}+\cdots+\alpha_{n} \leq s-\frac{n}{2}, \quad \alpha_{2} \leq \lambda_{2}-\frac{1}{2}, \cdots, \alpha_{n} \leq \lambda_{n}-\frac{1}{2}$,

$\mathscr{H} u(\alpha+\mathrm{i} \beta) \in L^{2}\left(\mathbb{R}^{n},\left(1+\| \beta_{1}||\right)^{\lambda_{1}} \cdots \cdot \mid\left(1+\left\|\beta_{n}\right\|\right)^{\lambda_{n}}\right)$, 
iii) $\mathscr{M u}$ is holomorphic in $\Omega_{s, s^{\prime}}$ and

$\mathscr{M u}(\alpha+\mathrm{i} \beta) \in L^{2}\left(\mathbb{R}^{n},\left(1+\left\|\beta_{1}\right\|\right)^{\lambda_{1}} \cdots \cdots\left(1+\left\|\beta_{n}\right\|\right)^{\lambda_{n}}\right)$ for the points $\alpha$ of the form $\alpha_{1}=s-\frac{1}{2}-\lambda_{2}-\cdots-\lambda_{n}, \alpha_{2}=\lambda_{2}-\frac{1}{2}, \cdots, \alpha_{n}=\lambda_{n}-\frac{1}{2}$ where

$0 \leq \lambda_{2}+\cdots+\lambda_{n} \leq s+s^{\prime}$.

Proof. From the operational properties of the Mellin transformation and Proposition 2 we obtain from (5) that for $|\lambda| \leq s+s^{\prime}$

$$
\begin{aligned}
& \mathscr{M}\left(x^{1 / 2} x_{1}^{-s+|\lambda|} D^{\lambda} u\right)(z) \\
= & p_{\lambda}(z) \mathscr{M u}\left(z_{1}+s-\frac{1}{2}-\lambda_{2}-\cdots-\lambda_{n}, z_{2}+\lambda_{2}-\frac{1}{2}, \cdots, z_{n}+\lambda_{n}-\frac{1}{2}\right)
\end{aligned}
$$

belongs to $L^{2}\left(\boldsymbol{R}^{n}\right)$ as a function of $\operatorname{Im} z$ for any fixed $\operatorname{Re} z$ such that

$$
\operatorname{Re} z_{1}+\operatorname{Re} z_{2}+\cdots+\operatorname{Re} z_{n} \leq 0, \quad \operatorname{Re} z_{2} \leq 0, \cdots, \operatorname{Re} z_{n} \leq 0
$$

where

$$
\begin{gathered}
p_{\lambda}(z)=\left(z_{1}+s-\frac{1}{2}-\lambda_{1}-\cdots-\lambda_{n}+1\right) \cdots\left(z_{1}+s-\frac{1}{2}-\lambda_{2} \cdots-\lambda_{n}\right) \\
\cdots \cdot\left(z_{n}+1-\frac{1}{2}\right) \cdots\left(z_{n}+\lambda_{n}-\frac{1}{2}\right)
\end{gathered}
$$

By changing the variable $z$ one can see that all assertions of Proposition 3 follow from those of Proposition 2.

Proposition 4. Let $(\stackrel{\circ}{x}, \stackrel{\circ}{\xi}) \in T_{0}^{*}\left(\boldsymbol{R}^{n}\right), \delta \stackrel{\circ}{x} \in \boldsymbol{R}_{+}^{n}, u \in S P(s,-\infty) . \quad u \in S P\left(s, s^{\prime}\right)$ 2-microlocally at the point $(\dot{x}, \xi, \delta \dot{x}, 0)$ if and only if there exist functions $\chi, \kappa, \rho$ satisfying conditions $1^{\circ}, 2^{\circ}, 3^{\circ}$ respectively, such that

$$
\left.\chi(z) \rho(z) \mathscr{M}(\kappa u)(z)\right|_{z=\alpha+\mathrm{i}} \cdot \in L^{2, s+s^{\prime}}\left(\boldsymbol{R}^{n}\right)
$$

for $\alpha_{1}+\cdots+\alpha_{n} \leq s-\frac{n}{2}$.

Corollary 2. The point $(\stackrel{\circ}{x}, \stackrel{\circ}{\xi}, \delta \dot{x}, 0)$ does not belong to $2 \mathrm{WF}^{S P(s, \infty)}$ if and only if for some $\chi, \kappa, \rho$ satisfying conditions $1^{\circ}, 2^{\circ}, 3^{\circ} \chi(z) \rho(z) \mathscr{M}(\kappa u)(z)$ as a function of $\operatorname{Im} z$ is rapidly decreasing for $z$ : $\operatorname{Re} z_{1}+\cdots+\operatorname{Re} z_{n} \leq s-\frac{n}{2}$.

\section{§ 3. Propagation of 2-Microlocal Singularities}

Theorem 1. (Propagation of singularities along the incoming bicharacteristic). Let $\delta \stackrel{\circ}{x}=(-1,0), \stackrel{\circ}{\xi}=\left(0, \stackrel{\circ}{\xi}^{\prime}\right)$ and let $v \in S P(s, \infty)$. Suppose that $v \in H^{s+\sigma}$ 
microlocally at $(x, \stackrel{\circ}{\xi})$ for every $x=\left(x_{1}, 0\right)$ where $x_{1}<0$ and that $w=\frac{\partial}{\partial x_{1}} v \in$ $S P(s-1, \sigma+1) 2$-microlocally at $(0, \dot{\xi}, \delta \dot{x}, 0)$. If $\sigma>-\frac{1}{2}$ then $v \in S P(s, \sigma)$ 2-microlocally at $(0, \stackrel{\circ}{\xi}, \delta \dot{x}, 0)$.

Proof. Replacing $v$ by $q(D) v$ where $q(D)$ is a suitable pseudodifferential

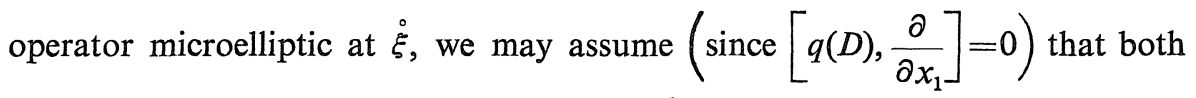
$v$ and $w$ are microlocally concentrated near $\stackrel{\circ}{\xi}$. Further we may multiply $v$ by a bump function $\varphi$ at zero and observe that $\frac{\partial}{\partial x_{1}}(\varphi v)=\left(\frac{\partial}{\partial x_{1}} \varphi\right) v+\varphi \frac{\partial}{\partial x_{1}} v$ satisfies the same assumption as $w$ since $\left(\frac{\partial}{\partial x_{1}} \varphi\right) v \in H^{s+\sigma}$ (by the assumption that $v \in H^{s+\sigma}$ microlocally at $\left(\left(x_{1}, 0\right), \stackrel{\circ}{\xi}\right)$ with $x_{1}<0$ we have $\left(\frac{\partial}{\partial x_{1}} \varphi\right) v \in S P(s, \sigma) \subset$ $S P(s-1, \sigma+1)$ because $\left.0 \notin \operatorname{supp}\left(\frac{\partial}{\partial x_{1}} \varphi\right)\right)$. Consequently we may assume that $v$ and $w$ are microlocally concentrated near $(0, \stackrel{\circ}{\xi})$. Finally changing $x_{1}$ to $-x_{1}$ we assume that $\delta \dot{x}=(1,0)$. Let $\tilde{\chi}$ be a cut-off function at $(1,0)$ subordinated to $\Gamma$ homogeneous of order zero (supp $\tilde{\chi} \subset \Gamma$ and $\tilde{\chi} \equiv 1$ on a cone $\Gamma_{1}$ tangent to $x$, slightly smaller then $\Gamma$ ) such that

$$
\tilde{w}=\tilde{\chi} w \in S P(s-1, \sigma+1) \text { globally. }
$$

First we show that there exists a unique Mellin distribution $u$ such that

$$
\tilde{w}=\frac{\partial}{\partial x_{1}} u \text {. }
$$

By Proposition $3 \mathscr{H} \tilde{w}(z)$ is holomorphic for $\sum_{1}^{n} \operatorname{Re} z_{j}<s-1-\frac{n}{2}, \operatorname{Re} z_{2}<s+\sigma$ $-\frac{1}{2}, \cdots, \operatorname{Re} z_{n}<s+\sigma-\frac{1}{2}$. Computing formally the Mellin transform of (8) we get

$$
\mathscr{M} \tilde{w}(z)=\left(z_{1}+1\right) \mathscr{M u}\left(z_{1}+1, z^{\prime}\right), \quad z^{\prime}=\left(z_{2}, \cdots, z_{n}\right)
$$

hence

$$
\mathscr{M} u(z)=\frac{\mathscr{M} \tilde{w}\left(z_{1}-1, z^{\prime}\right)}{z_{1}}
$$

on the set $\left\{\sum_{j} \operatorname{Re} z_{j}<s-\frac{n}{2}, \operatorname{Re} z_{2}<s+\sigma-\frac{1}{2}, \cdots, \operatorname{Re} z_{n}<s+\sigma-\frac{1}{2}, \operatorname{Re} z_{1}<0\right\}$.

Moreover for a fixed $\stackrel{\circ}{\alpha}$ such that $\sum \stackrel{\circ}{\alpha}_{j}<s-\frac{n}{2}, \stackrel{\circ}{\alpha}_{2}<s+\sigma-\frac{1}{2}, \cdots, \stackrel{\circ}{\alpha}_{n}<s+\sigma-\frac{1}{2}$, 
$\alpha_{1}<0$ we have from Proposition 3 (ii) that $\mathscr{M} \tilde{w}(\tilde{\alpha}-1+i \beta) \in L^{2}\left(\boldsymbol{R}^{n}\right)$. Since $\left|\stackrel{\circ}{\alpha}_{1}+\mathrm{i} \beta_{1}\right|>\left|\dot{\alpha}_{1}\right|>0$ it follows that also

$$
\frac{\mathscr{M} \tilde{w}(\stackrel{\circ}{\alpha}-1+\mathrm{i} \beta)}{\dot{\alpha}_{1}+\mathrm{i} \beta_{1}} \in L^{2}\left(\boldsymbol{R}^{n}\right) .
$$

Thus the inversion theorem for the Mellin transformation (see [2]) implies the existence and uniqueness of the desired Mellin distribution $u$.

From (ii) in Proposition 3 we get

$$
\mathscr{M} u(\alpha+\mathrm{i} \beta) \in L^{2}\left(\boldsymbol{R}^{n},\left(1+\left\|\beta_{1}\right\|\right)^{\lambda_{1}} \cdots \cdot\left(1+\left\|\beta_{n}\right\|\right)^{\lambda_{n}}\right)
$$

for every fixed $\alpha$ such that $\sum \alpha_{j} \leq s-\frac{n}{2}, \alpha_{2}<\lambda_{2}-\frac{1}{2}, \cdots, \alpha_{n}<\lambda_{n}-\frac{1}{2}, \alpha_{1}<0$, since as before $\left|\alpha_{1}+\mathrm{i} \beta_{1}\right| \geq\left|\alpha_{1}\right|>0$.

In order to retain the information on $u$ which will enable us to conclude that $u \in S P(s, \sigma)$ 2-microlocally at $\delta \dot{x}=(1,0)$ we have to assume, according to (iii) in Proposition 3, that

$$
\alpha_{1}=s-\frac{1}{2}-\lambda_{2}-\cdots-\lambda_{n}<0 \quad \text { for } \quad 0 \leq \lambda_{2}+\lambda_{3}+\cdots+\lambda_{n} \leq s+\sigma
$$

i.e. that $\sigma>-\frac{1}{2}$ and $s<\frac{1}{2}$ (see Fig. 1)

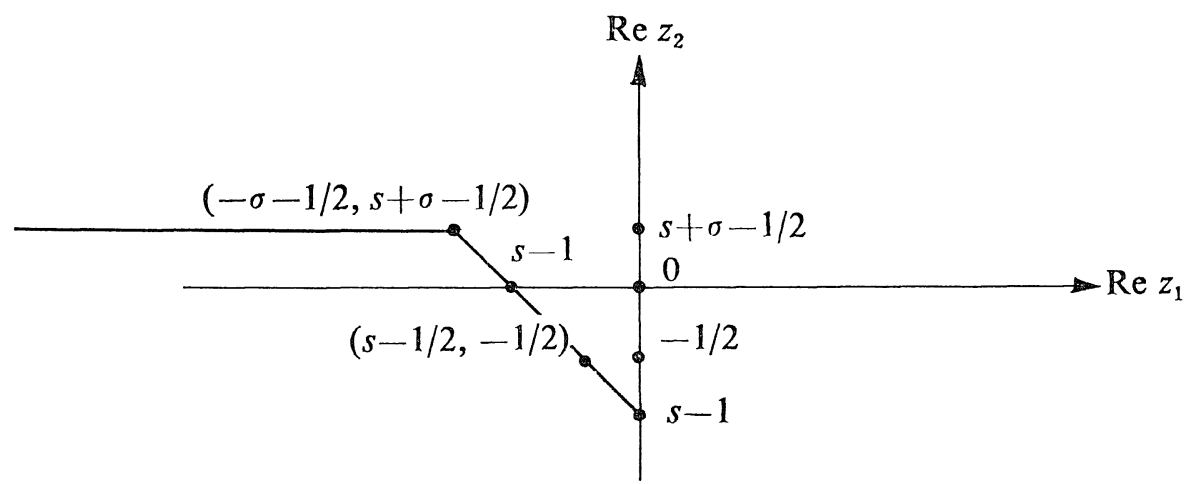

Fig. 1

Let $\Gamma_{1} \subset \Gamma$ be a cone tangent to the axis $x_{1}$ such that $\tilde{\chi} \equiv 1$ on $\Gamma_{1}$. We shall prove that $u=v$ on $\Gamma_{1}$.

We have

$$
\frac{\partial}{\partial x_{1}} u=\frac{\partial}{\partial x_{1}} v \quad \text { on } \quad \Gamma_{1}
$$


Hence for $x \in \Gamma_{1} u-v=g\left(x^{\prime}\right)$-a Mellin distribution on $\Gamma_{1}$ in variables $x^{\prime}=\left(x_{2}, \cdots, x_{n}\right)$. Since both $u$ and $v$ have bounded support and $\Gamma_{1}$ is tangent to the axis $x_{1}$ it follows that $g \equiv 0$ on $\Gamma_{1}$.

Let $\Gamma_{0}$ be the cone $\left\{x \in \mathbb{R}_{+}^{n}: 0<x_{1}<1, x_{2}<x_{1}, \cdots, x_{n}<x_{1}\right\}$ (see Fig. 2). We want to show that $\left.u\right|_{\Gamma_{0}} \in S P(s, \sigma)$. From Example 2 in [2] we know that if $\chi_{0}$ is the characteristic function of $\Gamma_{0}$, we have

$$
\mathscr{H} \chi_{0}(z)=\frac{(-1)^{n}}{\left(z_{1}+z_{2}+\cdots+z_{n}\right) z_{2} \cdots \cdots z_{n}} .
$$

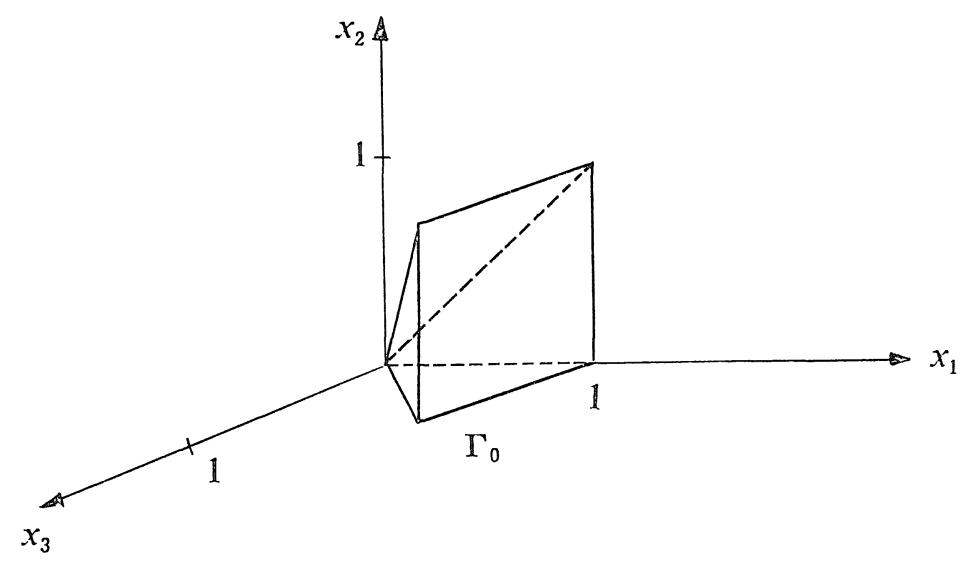

Fig. 2

Next (see [3], Proposition 5) for every $\stackrel{\circ}{\alpha}$ such that $\sum \stackrel{\circ}{\alpha}_{j} \leq s-\frac{n}{2}, \stackrel{\circ}{\alpha}_{2} \leq s+\sigma-\frac{1}{2}$, $\cdots, \stackrel{\circ}{\alpha}_{n} \leq s+\sigma-\frac{1}{2}, \stackrel{\alpha}{\alpha}_{1}<0$ we have

$$
\mathscr{H}\left(x_{0} u\right)(\dot{\alpha}+\mathrm{i} \beta)=\lim _{\substack{\alpha \rightarrow \dot{\alpha} \\ \alpha<<\infty}}\left(\mathscr{M} u(\dot{\alpha}+\mathrm{i} \gamma) * \mathscr{M}\left(\chi_{0}\right)(\alpha-\dot{\alpha}+\mathrm{i} \gamma)\right)(\beta)
$$

which, after the change of variables $\bar{\gamma}_{1}=\gamma_{1}+\cdots+\gamma_{n}, \bar{\gamma}_{2}=\gamma_{2}, \cdots, \bar{\gamma}_{n}=\gamma_{n}$ in the convolution, is the $n$-dimensional Hilbert transform of $\mathscr{M u}\left(\stackrel{\circ}{\alpha}+\mathrm{i}_{\bullet}\right)$. Now it is a well known fact that the Hilbert transform maps $L^{2}$-functions into $L^{2}$ functions. This proves that $\mathscr{M}\left(\chi_{0} u\right)(\stackrel{\alpha}{\alpha}+\mathrm{i} \beta) \in L^{2}$, and the same is true for $\mathscr{H}\left(x^{1 / 2} x_{1}^{-s+|\lambda|} \chi_{0} D^{\lambda} u\right)$ for $|\lambda| \leq s+\sigma$. This in view of Lemma 3 shows that $\left.u\right|_{\Gamma_{0}} \in S P(s, \sigma)$. Since $v=u$ on $\Gamma_{1}$ it follows that $\left.v\right|_{\Gamma_{1} \cap \Gamma_{0}} \in S P(s, \sigma)$.

Finally we note that the condition $s<\frac{1}{2}$ is not an essential restriction on $w$. Indeed, we may replace $w$ by $\Delta^{m} w$ for $m$ large enough where $\Delta$ is the Laplace 
operator in $\boldsymbol{R}^{n}$ and use the facts that $\left[\Delta^{m}, \frac{\partial}{\partial x_{1}}\right]=0, \Delta^{m}: S P\left(s, s^{\prime}\right) \rightarrow S P\left(s-2 m, s^{\prime}\right)$ and if $\Delta^{m} u \in S P\left(s, s^{\prime}\right), u \in S P(s,-\infty)$ then $u \in S P\left(s+2 m, s^{\prime}\right)$, since an operator elliptic in the classical sense is 2-microelliptic.

Lemma 4. Let $\Gamma$ be a proper cone in $\boldsymbol{R}_{+}^{n}$, and $\Gamma^{\prime}$ and open subcone in $\Gamma$. Write $x=\left(x_{1}, x^{\prime}\right)$ for points $x \in \boldsymbol{R}^{n}$ where $x^{\prime} \in \boldsymbol{R}^{n-1}$. Let $g\left(x^{\prime}\right)$ be a Mellin distribution on $\Gamma$, independent of $x_{1}$ and such that $g\left(x^{\prime}\right) \in S P\left(s, s^{\prime}\right)$ on $\Gamma^{\prime}$. Then $g\left(x^{\prime}\right) \in S P\left(s, s^{\prime}\right)$ on $\Gamma$.

Proof. Let $\chi^{\prime}$ be a cut-off function subordinated to $\Gamma^{\prime}$ such that $\chi^{\prime}(x) g\left(x^{\prime}\right) \in S P\left(s, s^{\prime}\right)$ globally. Since for every vector $\delta x \in \Gamma$ we can find $k>0$ such that

$$
\chi(x)=\chi^{\prime}\left(k x_{1}, x^{\prime}\right)
$$

is a cut-off function in the direction of $\delta x$, it is enough to prove that $\chi(x) g\left(x^{\prime}\right) \in$ $S P\left(s, s^{\prime}\right)$ globally. We have

$$
\begin{aligned}
\mathscr{M}\left(\chi(x) g\left(x^{\prime}\right)\right)\left(z_{1}, z^{\prime}\right) & =1_{x_{1}} \otimes g\left(x^{\prime}\right)\left[\chi(x) x_{1}^{-z_{1}-1} x^{\prime-z^{\prime}-1}\right] \\
& =g\left[x^{\prime-z^{\prime}-1} \int_{\boldsymbol{R}} \chi\left(x_{1}, x^{\prime}\right) x_{1}^{-z_{1}-1} d x_{1}\right] \\
& =k^{z_{1}} g\left[x^{\prime-z^{\prime}-1} \int_{\boldsymbol{R}} x^{\prime}\left(x_{1}, x^{\prime}\right) x_{1}^{-z_{1}-1} d x\right] \\
& =k^{z_{1}} \mathscr{M}\left(\chi^{\prime}(x) g\left(x^{\prime}\right)\right)(z),
\end{aligned}
$$

because

$$
\int_{\boldsymbol{R}} \chi^{\prime}\left(k x_{1}, x^{\prime}\right) x_{1}^{-z_{1}-1} d x_{1}=k^{z_{1}} \int_{\boldsymbol{R}} \chi^{\prime}\left(x_{1}, x^{\prime}\right) x_{1}^{-z_{1}-1} d x_{1} .
$$

Since $\left|k^{z_{1}}\right|=k^{\operatorname{Re} z_{1}}$ thus for a fixed $\operatorname{Re} z$ the multiplier $k^{z_{1}}$ has no influence on the behaviour of the Mellin transform as $|\operatorname{Im} z| \rightarrow \infty$. This in view of Proposition 3 ends the proof.

Theorem 2. (Propagation of singularities along second bicharacteristics). Let $\Gamma$ be a cone in $\boldsymbol{R}^{n}$ not tangent to $x_{1}$ i.e. such that $\bar{\Gamma} \cap\left\{\left(x_{1}, 0\right), x_{1} \in \boldsymbol{R}\right\}=\{0\}$. Suppose $v \in S P(-\infty,-\infty)$ on $\Gamma$ and let $\frac{\partial}{\partial x_{1}} v=w \in S P(s-1, \sigma+1) 2$-microlocally at the points $(0, \stackrel{\circ}{\xi}, \delta x, 0)$ for $\delta x \in \Gamma$ where $\stackrel{\circ}{\xi}=\left(0, \stackrel{\circ}{\xi}^{\prime}\right)$ for some fixed $\dot{\xi}^{\prime}$. If $v \in S P(s, \sigma)$ 2-microlocally at $(0, \xi, \delta x, 0)$ for $\delta x \in \Gamma^{\prime}-a$ subcone of $\Gamma$, then $v \in S P(s, \sigma) 2$-microlocally at $(0, \stackrel{\circ}{\xi}, \delta x, 0)$ for $\delta x \in \Gamma$. 
Proof. We assume that $u$ and $w$ are microlocally concentrated near $\stackrel{\circ}{\text {. }}$ Further (subject to a transformation of the form

$$
\left[\begin{array}{rr} 
\pm 1 & B \\
0 & A
\end{array}\right]
$$

where $A \in G L(n-1), B \in \mathbb{R}^{n-1}$, which preserves $\left.\frac{\partial}{\partial x_{1}}\right)$ we may assume that $\Gamma$ is a proper subcone of $\mathbb{R}_{+}^{n}$. Let $\tilde{\chi}$ be a cut-off function subordinated to $\Gamma$ (i.e. $\tilde{\chi} \in C^{\infty}\left(\boldsymbol{R}_{+}^{n}\right), \tilde{\chi}$ homogeneous of order zero, supp $\tilde{\chi} \subset \Gamma$ and $\tilde{\chi} \equiv 1$ on a slightly smaller proper subcone of $\Gamma$ ), and $\varphi$ a bump function at 0 such that $\tilde{w}=\varphi \tilde{\chi} w \in$ $S P(s-1, \sigma+1)$ globally.

Analogously to the proof of Theorem 1 we show that there exists a Mellin distribution $u$ such that $\tilde{w}=\frac{\partial}{\partial x_{1}} u$. Computing the Mellin transformation we get

$$
\mathscr{H} u(z)=\frac{\mathscr{M} \tilde{w}\left(z_{1}-1, z^{\prime}\right)}{z_{1}}
$$

and as in the proof of Theorem 1 we conclude in view of Corollary 2 that

$$
\mathscr{H} u(\alpha+\mathrm{i} \beta) \in L^{2, s+\sigma}\left(\mathbb{R}^{n}\right)
$$

for every fixed $\alpha$ such that $\sum \alpha_{j} \leq s-\frac{n}{2}, \alpha_{1}<0$.

We want to prove that $\mathscr{M} \chi u(\dot{\alpha}+\mathrm{i} \beta) \in L^{2}\left(\mathbb{R}^{n}\right)$ where $\dot{\alpha}$ is a fixed point satisfying $\sum \dot{\alpha}_{j}=s-\frac{n}{2}, \AA_{\alpha}<0$, and $\chi$ is the characteristic function of a proper cone containing $\Gamma$. To use the same technique as in the proof of Theorem 1 we suppose (again, by applying a transformation of the form $\left[\begin{array}{ll}1 & B \\ 0 & A\end{array}\right]$ ) that $\Gamma \subset\left\{x \in \boldsymbol{R}_{+}^{n}: x_{2}<x_{1}, \cdots, x_{n}<x_{1}\right\}$. We also suppose that supp $\tilde{w} \subset\left\{x \in \mathbb{R}_{+}^{n}:\right.$ $\left.x_{1} \leq 1\right\}$. For $x \in \Gamma$ we have $x_{1}<C_{2} x_{2}, \cdots, x_{1}<C_{n} x_{n}$ for some positive constants $C_{2}, C_{3}, \cdots, C_{n}$. Since $u\left(x_{1}, x^{\prime}\right)=-\int_{x_{1}}^{\infty} \tilde{w}\left(t, x^{\prime}\right) d t$ it follows that for $x \in \operatorname{supp} u x_{1}<C_{2} x_{2}, \cdots, x_{1}<C_{n} x_{n}$ and $x_{1} \leq 1$. Thus if $\chi^{0}$ is the characteristic function of the set $\Gamma_{1}^{0}=\left\{x \in \mathbb{R}_{+}^{n}: x_{1} \leq 1, x_{2}<x_{1}, \cdots, x_{n}<x_{1}\right\}$ it follows that supp $\chi^{0} u$ is a proper cone $\tilde{\Gamma}$ in $\mathbb{R}_{+}^{n}$ since $x_{j} \sim x_{k} j, k=1,2, \cdots, n$ on supp $\chi^{0} u$. The proof that $\mathscr{M} \chi^{0} u(\stackrel{\circ}{\alpha}+\mathrm{i} \beta) \in L^{2}\left(\mathbb{R}^{n}\right)$ is the same as in the proof of Theorem 1. Analogously, in view of Corollary 1 we get $u \in \operatorname{SP}(s, \sigma)$ on $\tilde{\Gamma}$. The final thing now is to compare $u$ and $v$. On the set where $\varphi \tilde{x} \equiv 1$ we have $\frac{\partial}{\partial x_{1}} u=\frac{\partial}{\partial x_{1}} v$ 
thus $u-v=g\left(x^{\prime}\right)$ is a Mellin distribution on that set depending only on $x^{\prime}$. Since on a smaller subcone $v \in \operatorname{SP}(s, \sigma)$ it follows that $g\left(x^{\prime}\right) \in S P(s, \sigma)$ on that subcone. Now we apply Lemma 4 to get $g\left(x^{\prime}\right) \in S P(s, \sigma)$ on $\Gamma$. This ends the proof.

Theorem 3. (Propagation of singularities along the outgoing bicharacteristic). Let $\delta \dot{x}=(1,0), \dot{\xi}=\left(0, \dot{\circ}^{\prime}\right)$ and let $v \in S P(s,-\infty)$ be such that $v=\operatorname{SP}(s, \sigma)$ 2-microlocally at the points $(0, \stackrel{\circ}{\xi}, \delta x, 0)$ where $\delta x \in \boldsymbol{R}_{+}^{n}$. Suppose that

$$
w=\frac{\partial}{\partial x_{1}} v
$$

and $w \in S P(s-1, \sigma+1)$ 2-microlocally at $(0, \xi, \delta \dot{x}, 0)$. If $\sigma<-\frac{1}{2}$ then $v \in \operatorname{SP}(s, \sigma) 2$-microlocally at $(0, \dot{\xi}, \delta \dot{x}, 0)$.

Proof. By applying to $v$ a suitable cut-off function $\tilde{\chi}$ at $(1,0)$ subordinated to a cone $\Gamma \subset \boldsymbol{R}_{+}^{n}$ tangent only to $x_{1}$, and a cut-off function $\rho$ in the direction $\dot{\xi}$ we may assume that supp $v \subset \Gamma$ and $w \in S P(s-1, \sigma+1)$ locally at zero. Thus for a suitable bump function $\varphi$ at zero $\tilde{w}=\varphi w \in S P(s-1, \sigma+1)$ globally.

As in the proof of Theorem 1 we prove the existence (and uniqueness) of a Mellin distribution $u$ such that

$$
\tilde{w}=\frac{\partial}{\partial x_{1}} u
$$

Moreover

$$
\mathscr{M} u(z)=\frac{H(z)}{z_{1}} \quad \text { for } \quad \operatorname{Re} z_{1}<0, \operatorname{Re} z \in A
$$

where $H(z)=\mathscr{H}\left(x_{1} \tilde{w}\right)(z)$ is holomorphic on the set $A+\mathrm{i} \boldsymbol{R}^{n}$ and

$$
A=\left\{\alpha \in \boldsymbol{R}^{n}: \sum \alpha_{j}<s-\frac{n}{2}, \alpha_{2}<s+\sigma-\frac{1}{2}, \cdots, \alpha_{n}<s+\sigma-\frac{1}{2}\right\} .
$$

Further for points $\alpha \in \bar{A}, H(\alpha+\mathrm{i} \beta) \in L^{2}\left(\boldsymbol{R}^{n}\right)$ as a function of $\beta$. We want to invert $F(z)=H(z) / z_{1}$ at a point $\stackrel{\circ}{\alpha} \in A$ such that $\stackrel{\circ}{\alpha}_{1}>0$. To this end we use the generalized Mellin transformation $M^{\circ}$ introduced in [3]. We have

$$
\left(M^{\stackrel{\circ}{\alpha}} f\right)(\beta)=F(\stackrel{\circ}{\alpha}+\mathrm{i} \beta)
$$

where

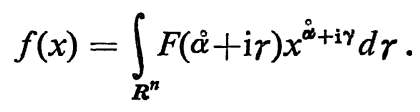


Take a point $\tilde{\alpha}=\left(\widetilde{\alpha}_{1}, \stackrel{\circ}{\alpha}^{\prime}\right)$ where $\widetilde{\alpha}_{1}<0, \stackrel{\circ}{\alpha}=\left(\dot{\alpha}_{1}, \stackrel{\circ}{\alpha}^{\prime}\right)$. Then

$$
u(x)=\int_{\boldsymbol{R}^{n}} F(\tilde{\alpha}+\mathrm{i} \gamma) x^{\tilde{\alpha}+\mathrm{i} \gamma} d \gamma .
$$

Fixing $\dot{\alpha}^{\prime}+\mathrm{i} \gamma^{\prime}$ and applying the residuum theorem in variable $z_{1}$ we get

$$
\int_{\boldsymbol{R}} F(\widetilde{\alpha}+\mathrm{i} \gamma) x_{1^{\tilde{\alpha}}+\mathrm{i} \gamma_{1}} d \gamma_{1}=\int_{\boldsymbol{R}} F(\stackrel{\circ}{\alpha}+\mathrm{i} \gamma) x_{1}^{\stackrel{\circ}{\alpha}+\mathrm{i} \gamma_{1}} d \gamma_{1}+H\left(0, \stackrel{\circ}{\alpha}^{\prime}+\mathrm{i} \gamma^{\prime}\right) .
$$

Thus we find

$$
u(x)=f(x)+\int_{\boldsymbol{R}^{n-1}} H\left(0, \alpha^{\prime}+\mathrm{i} \gamma^{\prime}\right) x^{\prime \alpha^{\prime}+\mathrm{i} \gamma^{\prime}} d \gamma^{\prime} .
$$

Since by the definition of the Mellin transformation

$$
H\left(0, \stackrel{\circ}{\alpha}+\mathrm{i} \gamma^{\prime}\right)=x_{1} \tilde{w}\left[x_{1}^{-1} x^{\prime-\alpha^{\prime}-\mathrm{i} \gamma^{\prime}-1}\right]=\tilde{w}\left[x^{\prime-\dot{\alpha}^{\prime}-\mathrm{i} \gamma^{\prime}-1}\right],
$$

we have by the inversion rule (9)

$$
\begin{aligned}
\int_{\boldsymbol{R}^{n-1}} H\left(0, \alpha^{\prime}+\mathrm{i} \gamma^{\prime}\right) x^{\prime \alpha^{\prime}+\mathrm{i} \gamma^{\prime}-1} d \gamma^{\prime} & =\int_{\boldsymbol{R}} \tilde{w}\left(x_{1}, x^{\prime}\right) d x_{1} \\
& =\int_{\boldsymbol{R}} \frac{\partial}{\partial x_{1}} u\left(x_{1}, x^{\prime}\right) d x_{1}=-u\left(0, x^{\prime}\right)
\end{aligned}
$$

because $u$ has bounded support as a Mellin distribution. Thus

$$
f(x)=u(x)-u\left(0, x^{\prime}\right)
$$

Since $f(x)=u(x)-u\left(0, x^{\prime}\right)=\int_{0}^{x_{1}} w\left(t, x^{\prime}\right) d t$ we see that supp $f \subset \Gamma$. To prove that $f \in S P(s, \sigma)$ locally we have to ensure analogously to the proof of Theorem 1 that

$$
\alpha_{1}=s-1 / 2-\lambda_{2}-\cdots-\lambda_{n}>0 \text { for } 0 \leq \lambda_{2}+\lambda_{3}+\cdots+\lambda_{n} \leq s+\sigma
$$

i.e. that $\sigma<1 / 2$ and $s>1 / 2$. Now multiplying $f$ by the characteristic function of the unit cube $\{0<x \leq 1\}$, which under the Mellin transformation amounts to computing the Hilbert transform of $M^{\AA} f\left(\right.$ since $\left.\mathscr{M} \chi(z)=\frac{(-1)^{n}}{z_{1} \cdots \cdot z_{n}}\right)$, we conclude, as in the proof of Theorem 1 , that $f \in S P(s, \sigma)$ locally.

Finally we compare $f$ and $v$. We have $\frac{\partial}{\partial x_{1}} f-\frac{\partial}{\partial x_{1}} v=0$ for $x \in \mathbb{R}_{+}^{n}, 0<$ $x_{1}<\tau$ for some $\tau$, thus $f-v=g\left(x^{\prime}\right)$ for $0<x_{1}<\tau$. Since $\operatorname{supp} f \operatorname{supp} v \subset \Gamma$ it 
follows that $g\left(x^{\prime}\right) \equiv 0$. This proves that $v \in S P(s, \sigma)$ locally at zero if $s>1 / 2$. The case $s \leq 1 / 2$ is reduced to the above by a similar reasoning as at the end of the proof of Theorem 1 .

Theorems 1, 2, 3 together give the following Bony's theorem on the propagation of 2-microlocal singularities:

Theorem 4. Let $\stackrel{\circ}{\xi}=\left(0, \dot{\circ}^{\prime}\right)$ and suppose $v \in S P(s,-\infty)$ microlocally at $(0, \dot{\xi})$ and $v \in H^{s-1 / 2}$ microlocally at $\left(x_{1}, 0, \stackrel{\circ}{\xi}\right)$ where $x_{1}<0$. If $w=\frac{\partial}{\partial x_{1}} v \in S P(s-1,-1 / 2)$ microlocally at $(0, \stackrel{\circ}{\xi})$ then $v \in S P(s-\varepsilon,-1 / 2)$ microlocally at $(0, \stackrel{\circ}{\xi})$ for any $\varepsilon>0$.

Proof. To apply Theorem 1 we observe that for $a \in \mathbb{R} S P(a,-1 / 2) \subset$ $S P(a-\varepsilon,-1 / 2+\varepsilon)$, while the inclusions $S P(a,-1 / 2) \subset S P(a,-1 / 2-\varepsilon) \subset$ $S P(a-\varepsilon,-1 / 2)$ allow us to apply Theorem 3 .

Concluding remark. The technique of the Mellin transformation presented in the paper can also be applied to get analogous results for the spaces $H^{s, s^{\prime}}$.

\section{References}

[1] Bony, J.M., Second microlocalization and propagation of singularities for semilinear hyperbolic equations, Hyperbolic equations and related topics (Katata/Kyoto, 1984), Academic Press, Boston, (1986), 11-49.

[2] Ziemian, B., The Mellin transformation and multidimensional generalized Taylor expansions of singular functions, J. Fac. Sci. Univ. Tokyo, 36 (1989), 263-295.

[3] Ziemian, B. and Szmydt, Z., Local existence and regularity of solutions of singular elliptic operators on manifolds with corner singularities, J. Diff. Equ., 83 (1990), 1-25. 
